\title{
Erratum to: Interactions Between Chronic Stress and Chronic Consumption of Caffeine on the Enzymatic Antioxidant System
}

\author{
Cristie Grazziotin Noschang - Rachel Krolow • Leticia Ferreira Pettenuzzo • \\ Mônica Colpini Ávila • Andrelisa Fachin • Danusa Arcego • Eduardo von Pozzer Toigo • \\ Leonardo Machado Crema $\cdot$ Luísa Amália Diehl $\cdot$ Deusa Vendite $\cdot$ Carla Dalmaz
}

Published online: 12 December 2009

(C) Springer Science+Business Media, LLC 2009

Erratum to: Neurochem Res (2009) 34:1568-1574

DOI 10.1007/s11064-009-9945-4

The author regrets an error occurred in Figs. 1c, 2c, 3c of the article, wherever the GPx activity is presented as pmol
NADPH oxidized/min/mg protein, the activity should read as nmol NADPH oxidized/min/mg protein.

The online version of the original article can be found under doi:10.1007/s11064-009-9945-4.

C. G. Noschang $(\bowtie) \cdot$ R. Krolow - L. F. Pettenuzzo •

M. C. Ávila · A. Fachin · D. Arcego · E. von Pozzer Toigo ·

L. M. Crema · L. A. Diehl · D. Vendite · C. Dalmaz

Departamento de Bioquimica, Instituto de Ciências Básica da Saúde, ICBS, UFRGS, Ramiro Barcellos, 2600, anexo., Porto Alegre, RS 90035-003, Brazil

e-mail: cristienoschang@hotmail.com

C. Dalmaz

e-mail: carladalmaz@yahoo.com.br

C. G. Noschang · R. Krolow · C. Dalmaz

Programa de Pós-Graduac,ão em Bioquimica, Instituto de Ciências Básica da Saúde, ICBS, UFRGS, Ramiro Barcellos, 2600, anexo., Porto Alegre, RS 90035-003, Brazil

L. M. Crema · L. A. Diehl · C. Dalmaz

Programa de Pós-Graduac,ão em Neurociências, Instituto de Ciências Básica da Saúde, ICBS, UFRGS, Ramiro Barcellos, 2600, anexo., Porto Alegre, RS 90035-003, Brazil 